\title{
Highly-nonlinear Chalcogenide Glass Devices for High-speed Signal Processing and Characterization
}

\author{
M.D. Pelusi ${ }^{1}$, T.D. Vo ${ }^{1}$, F. Luan ${ }^{1}$, S.J. Madden ${ }^{2}$, D.-Y. Choi ${ }^{2}$, D.A.P. Bulla ${ }^{2}$, B. Luther-Davies ${ }^{2}$, and B.J. Eggleton ${ }^{1}$ \\ ${ }^{1,2}$ ARC Centre for Ultrahigh bandwidth Devices for Optical Systems (CUDOS) \\ Phone: +61-2-9351-7697, Fax:+61-2-9351-7726, Email: m.pelusi@physics.usyd.edu.au \\ ( ${ }^{1}$ School of Physics, University of Sydney, New South Wales 2006, Australia) \\ ( ${ }^{2}$ Laser Physics Centre, Australian National University, Canberra ACT 0200, Australia)
}

\begin{abstract}
We review the latest advances in dispersion-shifted Chalcogenide waveguides enabling highly nonlinear and low dispersion planar rib circuits of centimetre length. Its performance advantages for more broadband and higher speed nonlinear signal processing are shown.
\end{abstract}

\section{Introduction}

All-optical signal processing is a key technology for enabling various functions applicable to future highspeed optical communications such as optical switching, wavelength conversion [1], and performance monitoring [2]. These can be performed on an ultra-fast timescale by harnessing the optical Kerr effect of an optical waveguide exhibiting a $\chi^{(3)}$ nonlinearity, whereby its refractive index changes in proportional to the instantaneous optical intensity, and the nonlinear refractive index, $n_{2}$. This gives rise to the nonlinear effects of cross-phase modulation (XPM) and four-wave mixing (FWM), which can underpin many all-optical signal processing functions [1]. The efficiency however, depends on the waveguide nonlinearity coefficient determined by $\gamma=(2 \pi / \lambda) \cdot\left(n_{2} / A_{\text {eff }}\right)$, where $A_{\text {eff }}$ is the effective mode area at the signal wavelength, $\lambda$. The nonlinear phase shift also scales with waveguide length which dictates the need for kilometers of standard single mode fiber (SMF), whose $\gamma$ is low, or hundreds of meters for silica based highly nonlinear fiber (HNF) [3].

Recent advances in fabricating planar rib waveguides from high index glasses such as silicon [4] and Chalcogenide (ChG) [1] have shown the capability for reaching much higher $\gamma$ through their combined high $n_{2}$ and small $A_{\text {eff }}$ allowing much shorter circuits on the order of centimeters. This translates to reduced dispersion effects, which otherwise degrades the FWM and XPM efficiency through the phase and group velocity mismatch respectively between co-propagating waves.

In this paper, we review the development of a dispersion-shifted planar rib waveguide based on the $\mathrm{ChG}$ glass, $\mathrm{As}_{2} \mathrm{~S}_{3}$ whose $n_{2}=3 \times 10^{-18} \mathrm{~m}^{2} / \mathrm{W}$ (i.e. $\approx 100$ times of silica). Its design for a smaller $A_{\text {eff }}$ produces both an increased $\gamma$ and tailoring of the net dispersion parameter, $D$, to near zero at the $1550 \mathrm{~nm}$ wavelength.

Its performance advantage for both wavelength conversion of $40 \mathrm{~Gb} / \mathrm{s}$ signals across the S-C-L optical bands of the optical communication spectrum, and high- speed time-division demultiplexing of $320 \mathrm{~Gb} / \mathrm{s}$ signals are shown. The photonic chip is also applied to an RF spectrum analyzer system enabling characterization and dispersion monitoring of $320 \mathrm{~Gb} / \mathrm{s}$ data signals.

\section{Waveguide characteristics}

The primary advance over previously reported $\mathrm{As}_{2} \mathrm{~S}_{3}$ planar rib waveguides is its design for higher $\gamma$ and tailoring of $D$ toward zero at the $1550 \mathrm{~nm}$ wavelength. This was realized by thinning the $\mathrm{As}_{2} \mathrm{~S}_{3}$ film during deposition [5], by nearly a factor of 3 to $0.85 \mu \mathrm{m}$. Photolithography and dry-etching [5] produced ribs as narrow as $2 \mu \mathrm{m}$ with a reduced $A_{\text {eff }}$ approaching $1 \mu \mathrm{m}^{2}$. This increased both $\gamma$ and waveguiding dispersion which has a sign countering the large material component of $\mathrm{As}_{2} \mathrm{~S}_{3}$ (=-364 ps/nm/km at $1550 \mathrm{~nm}$ wavelength). A typical device schematic is shown in Fig. 1. Waveguides

(a)

(b)
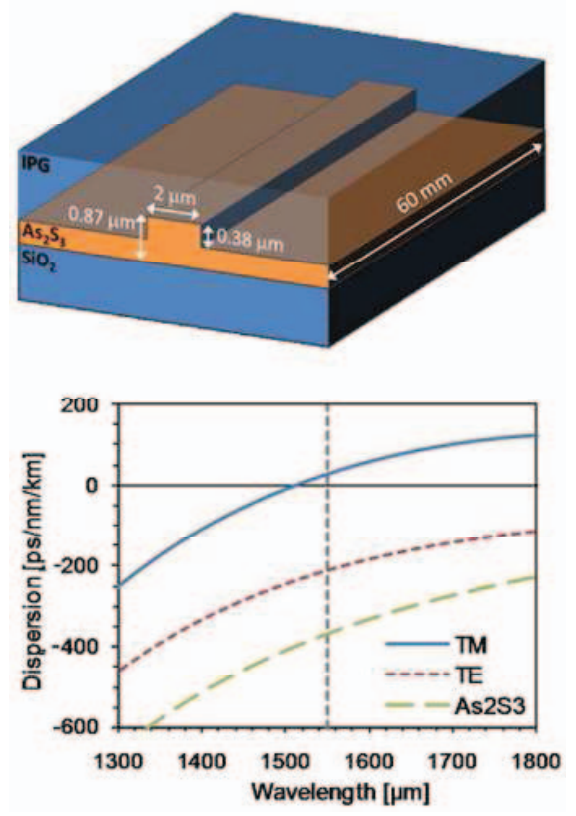

Fig. 1. a) Device illustration of a $6 \mathrm{~cm}$ length planar rib $\mathrm{As}_{2} \mathrm{~S}_{3}$ waveguide and (b) calculated group velocity dispersion of fundamental TM and TE modes compared to material dispersion of $\mathrm{As}_{2} \mathrm{~S}_{3}$ highlighting the dispersion-shifted design enabled by the thinner $\mathrm{As}_{2} \mathrm{~S}_{3}$ film thickness, as reported in [6]. 
with $\gamma$ as high as $9900 \mathrm{~W}^{-1} \mathrm{~km}^{-1}$ (more than quadruple that of previous $\mathrm{As}_{2} \mathrm{~S}_{3}$ chips and over 6000 times larger than standard single mode fiber) were produced for the narrowest $2 \mu \mathrm{m}$ wide rib. Furthermore, the enhanced waveguiding dispersion of the TM mode reduced $D$ by over an order of magnitude to as low as $28 \mathrm{ps} / \mathrm{nm}$. $\mathrm{km}$ (in the anomalous regime) as shown in Fig. 1(b).

The propagation loss for the waveguide TM mode varied between 0.2 to $0.65 \mathrm{~dB} / \mathrm{cm}$ for rib widths of 4 and $2 \mu \mathrm{m}$ respectively. This was more than previous (thicker film) $\mathrm{As}_{2} \mathrm{~S}_{3}$ rib waveguides [1] due to the increased mode penetration of the core/cladding interface, which enhances the scattering losses associated with its surface roughness. Coupling to SMF was achieved by lensed fibers of $2.5 \mu \mathrm{m}$ spot diameter which raised the device insertion loss by a further $\approx 3$ to $5 \mathrm{~dB}$ per facet for 4 and $2 \mu \mathrm{m}$ wide ribs respectively. This can be improved by incorporating on-chip tapers as reported for silicon [4].

\section{Broadband wavelength conversion}

All-optical wavelength conversion is a key function for enabling optically transparent communications networks with fewer OEO conversions. One approach is to use either XPM or FWM of the signal co-propagating through a nonlinear optical waveguide with a cw laser acting as a probe tuned to the desired converted signal wavelength. Optically filtering of either the XPM sideband or the FWM idler spectrum recovers a replica of the signal at a different wavelength. This has typically required hundreds of metres of HNF to achieve sufficient nonlinear phase shift [1]. Consequently, the maximum wavelength conversion range has been limited by dispersion effects due to the increased phase and group velocity mismatch between the co-propagating waves as their wavelength separation is increased.

The performance advantage of using dispersionshifted ChG waveguide was highlighted by wavelength conversion of a $40 \mathrm{~Gb} / \mathrm{s}$ return-to-zero signal over an $80 \mathrm{~nm}$ wavelength range [6]. The signal used a $2 \mathrm{ps}$ pulse width compatible with a $160 \mathrm{~Gb} / \mathrm{s}$ bit rate, and the total average power at the waveguide input connector was $100 \mathrm{~mW}$. Despite using such short pulses, the FWM and XPM observed in the optical spectrum at the output of the waveguide showed little variation with wavelength when tuning the $\mathrm{cw}$ laser from 1545 to $1570 \mathrm{~nm}$. Bit error rate measurements of the optically filtered idler from FWM showed error free performance.

\section{High-speed time-division demultiplexing}

All optical gating of a signal provides a means of timedivision demultiplexing a signal of bit rate, $B_{0}$, into its $N$ lower bit-rate tributaries even when $B_{0}$ is beyond the conventional $40 \mathrm{~Gb} / \mathrm{s}$ switching speed of opto-electronic modulators. This can be performed by co-propagating the signal with a synchronized optical pulse train of repetition $B_{0} / N$. This acts as a pump such that its coincidence with each signal pulse generates a new pulse by FWM, allowing its extraction by optical filtering [1]
Realizing this on a planar platform rather than with multiple optical fibers allows parallel demultiplexing of all channels on a single photonic integrated circuit. As a preliminary step in this direction, all-optical timedivision demultiplexing of a single $10 \mathrm{~Gb} / \mathrm{s}$ tributary from a $320 \mathrm{~Gb} / \mathrm{s}$ signal was demonstrated by FWM in a $6 \mathrm{~cm}$ length waveguide. The $320 \mathrm{~Gb} / \mathrm{s} \mathrm{RZ}$ signal at the $1561 \mathrm{~nm}$ wavelength was combined with a $10 \mathrm{GHz}$ pulse train of 2 ps duration at $1550 \mathrm{~nm}$ wavelength. The total average power at the waveguide input connector was $100 \mathrm{~mW}$. The spectrum at the waveguide output revealed an idler pulse at $1539 \mathrm{~nm}$ produced by FWM, which was subsequently filtered to extract the $10 \mathrm{~Gb} / \mathrm{s}$ tributary.

\section{Broadband RF spectrum analysis}

The performance advantages of dispersion-shifted $\mathrm{ChG}$ waveguides are emphasized when applied to an alloptical RF spectrum analyser [2]. This is a technique for capturing the power spectrum of the signal's intensity (rather than its electric field as measured on an optical spectrum analyser (OSA)). The original demonstration in HNF [7], showed the possibility for a measurement bandwidth exceeding an order of magnitude greater than conventional opto-electronic techniques (using a highspeed photodetector with electrical spectrum analyser). The implementation is conceptually the same as for the all-optical wavelength converter described above, except it uses a lower signal power to ensure a phase shift «1 radian so that the XPM sidebands observed an OSA correspond to the power spectrum of the signal intensity.

While original experiments using HNF reported a bandwidth of $\approx 800 \mathrm{GHz}$ [7], the use of the dispersionshifted ChG waveguide has demonstrated both a broader multi-terahertz measurement bandwidth and distortionfree trace even for very short optical pulses [2]. Furthermore, "colorless" operation is achieved over a broader ranging signal wavelength. Its effectiveness for characterizing $320 \mathrm{~Gb} / \mathrm{s}$ signal and features not evident from a conventional OSA were highlighted along with its powerful capability for dispersion monitoring.

\section{Conclusions}

Dispersion-shifted Chalcogenide waveguides designed for combined high nonlinearity and low dispersion show significant performance advantages for broadband signal processing in terms of a wider wavelength tuning range and reduced signal distortion. These, together with the monolithic structure capable of integrating multiple functions on a single chip, highlight its potential for application to future high-speed optical communications.

\section{References}

1. M.Pelusi et al, IEEE J Sel. Top. Quant. 14 (2008) p.529

2. M. Pelusi et al, Nature Photon. 3 (2009) p. 139

3. M. Takahashi et al, J. Lightwave Technol. 23 (2005) p. 3615

4. M. Foster et al, Nature Photon., 456 (2008) p. 81

5. S. Madden et al, Opt. Express, 15 (2007) p. 14414

6. F. Luan et al, Opt. Express, 17 (2009) p. 3514

7. C. Dorrer et al, J. Lightwave Technol., 22 (2004) p. 266 